\title{
MANIPULACIÓN DE LA OVULACIÓN DEL FOLÍCULO DOMINANTE CON PROSTAGLANDINAEN DIFERENTES ESTADIOS DEL CICLO ESTRUAL SOBRE LAS TASAS REPRODUCTIVAS EN OVINOS CORRIEDALE
}

\author{
Martín Urviola S. ${ }^{1}$, Víctor Leyva V. $^{2}$, Héctor Huamán U. ${ }^{3}$ y Wilber García $V^{4}$.
}

\section{Abstract}

Sixty-five 3-4 years old Corriedale ewes were used from April to August 2002 to evaluate the effect of $\mathrm{PGF}_{2 \propto}$ treatment at days 4 (GP4) and 10 (GP10) of the estrous cycle on (i) the percentage of estrus, (ii) variability within animals in the onset of estrus, (iii) the length of the treatment-estrus interval and, (iv) the rate of pregnancy. The estrous cycle was synchronized inserting intravaginal sponges impregnated with $30 \mathrm{mg}$ of fluorogestone acetate (FGA) during 12 days. After the occurence of estrus (estrus = day 0), 20 ewes at day 4 (GP4) and 22 at day 10 (GP10) were treated with $0.175 \mathrm{mg}$ of an analogue of PGF ${ }_{2 \propto}$. The 23 remaining ewes were not treated and kept as control group (GC). Estrus was detected by introducing vasectomized rams to the flock at 6,12 and $17 \mathrm{~h}$ on daily basis. All FGA-synchronizated ewes showed estrus, as well as after the treatment with PGF $_{2 \propto}$; however, the onset of estrus was significantly $(\mathrm{p} \leq 0.01)$ earlier in GP4 (34:28 h) and GC $(40: 16 \mathrm{~h})$ than in GP10 (47:45 h). GP4 presented smaller variability among ewes $(\mathrm{sd}=4: 26)$ in the length of the treatment-estrus interval than GP10 (sd=7:06). Pregnancy rate (no return to estrus) at day 17 after breeding were similar in groups GP10 (77.3\%) and GP4 (80.0\%), but slightly higher in GC $(91.3 \%)$. Pregnancy rate decreased by day 28 in all groups (63.6, 65.0 and $87.0 \%$, respectively), and remaining like that until day 72 , with the exception of GC (78.3\%).

Key words: ewe, estrous cycle, prostaglandin, synchronization

\section{RESUMIEN}

Se utilizaron 65 borregas Corriedale de 3 y 4 años de edad en los meses de abril a agosto del 2002 para evaluar el efecto de la administración de PGF $_{2 \propto}$ en los días 4 y 10 del ciclo estrual sobre (i) la incidencia del celo, (ii) la variabilidad entre animales en la presentación del celo, (iii) el intervalo tratamiento-celo y (iv) la tasa de preñez postservicio. El ciclo estrual fue sincronizado previamente con la inserción intravaginal por 12 días de esponjas impregnadas con $30 \mathrm{mg}$ de acetato de fluorogestona (FGA). Luego del celo

\footnotetext{
${ }^{1}$ Facultad de Medicina Veterinaria y Zootecnia, Universidad Nacional del Altiplano, Puno

${ }^{2}$ Laboratorio de Reproducción Animal, FMV-UNMSM. E-mail: vleyva@vet.unmsm.edu.pe

${ }^{3}$ Laboratorio de Bioquímica, Nutrición y Alimentación Animal, FMV-UNMSM

${ }^{4}$ Estación Experimental del Centro de Investigaciones IVITA-La Raya, FMV-UNMSM
} 
postsincronización (celo = día 0 ) se administró $0.175 \mathrm{mg}$ de un análogo de PGF $_{2}$ ? a 20 animales en el día 4 del ciclo estrual (GP4) y a 22 animales en el día 10 (GP10), en tanto que 23 animales quedaron sin tratamiento como grupo control (GC) para fines comparativos. Se detectó el celo con carneros vasectomizados a las 6,12 y $17 \mathrm{~h}$ de cada día. El $100 \%$ de las borregas presentó celo a la sincronización con $\mathrm{FGA}$ y con el tratamiento con $\mathrm{FGF}_{2 \text { ? }}$; sin embargo, el celo ocurrió significativamente (p? 0.01) más temprano en GP4 (34:28 h) y GC $(40: 16$ h) que en GP10 (47:45 h). GP4 presentó menor variabilidad entre borregas $(\mathrm{de}=4: 26)$ en el intervalo entre el tratamiento con PGF $_{2}$ ? y la presentación de celo, que GP10 (de=7:06). La tasa de preñez (no retorno al celo) a los 17 días postservicio fue similar en ambos grupos tratados con $\mathrm{PGF}_{2 \text { ? }}$ y ligeramente mayor en GC (77.3, 80.0 y 91.3\% para GP10, GP4 y GC, respectivamente), reduciéndose esta tasa a los 28 días en todos los grupos (63.6, 65.0 y $87.0 \%$, respectivamente) y manteniéndose hasta los 72 días con excepción de GC $(78.3 \%)$.

Palabras clave: borrega, ciclo estrual, prostaglandina, sincronización

\section{INTRODUCCIÓN}

Una de las principales limitaciones en el manejo reproductivo de ovinos es la deficiente detección y sincronización del celo en borregas, debido a la gran variabilidad que existe entre animales (Illera, 1994). El desarrollo de los métodos de control artificial del ciclo estrual se ha basado principalmente en el conocimiento sobre los mecanismos de control hormonal del ciclo estrual dirigidos a mejorar la eficiencia reproductiva. Paralelamente, se han desarrollado eficientes análogos de hormonas con acciones biológicas más potentes que las naturales. En ovinos, el desarrollo de estos métodos ha permitido la manipulación eficiente del celo y la ovulación para determinar el tiempo óptimo de la inseminación artificial, sincronizando el empadre y la parición a fin de permitir el establecimiento de programas apropiados de mejoramiento genético (Cárdenas, 1997).

Por lo general, estos métodos se basan en el control de la vida del cuerpo lúteo (CL) y consecuentemente de la actividad folicular (Cárdenas, 1997), a través de hormonas como la $\mathrm{PGF}_{2}$, identificada como el factor luteolítico del CL (McCracken et al., 1972). La identificación y manipulación de su es- tructura química ha permitido el desarrollo de hormonas análogas sintéticas, usadas mayormente para inducir la regresión del CL y consecuentemente el retorno al celo de las ovejas. El mayor efecto luteolítico de la $\mathrm{PGF}_{2}$ ? se observa en CL maduros (Acritopoulou y Haresign, 1980), estando reducido o ausente en CL ? 4 días de desarrollo (Acritopoulou y Haresign, 1980; Wiltbank y Niswender, 1992); sin embargo, existen reportes de un efecto luteolítico en CL de tres días (Rubianes et al., 1997a,b).

Se reporta que ovejas que responden a la $\mathrm{PGF}_{2 \text { ? }}$ presentan una gran variabilidad en el intervalo al celo entre animales, lo que evitaría un uso más extenso de esta hormona, particularmente, si la sincronización del estro se usa asociada con la inseminación artificial. Esta variabilidad puede ser debido al estado de desarrollo de los folículos preovulatorios en relación a la fase del ciclo estrual al momento de la sincronización, tal como ha sido observado en vacas con relación al crecimiento del folículo dominante (Savio et al., 1988; Ginther et al., 1989). Así, Champa (2000) sugiere que la sincronización de la ocurrencia de celo y ovulación es menos variable cuando se realiza durante la fase luteal temprana que durante la fase luteal avanzada. Por lo expuesto, se consideró 
como objetivos del presente estudio evaluar el efecto de la administración de la $\mathrm{PGF}_{2 \propto}$ en los días 4 y 10 del ciclo estrual de la oveja sobre la incidencia del celo, variabilidad entre animales en la presentación del celo y el intervalo entre tratamiento y el celo y su repercusión en la tasa de preñez.

\section{MateRIALes y Métodos}

El trabajo se realizó en el Centro de Investigación y Producción Chuquibambilla de la Universidad Nacional del Altiplano, ubicado en el departamento de Puno, a $14^{\circ}$ 50' 18" latitud sur, 70 44' 42" longitud oeste, y a una altitud de 3,974 $\mathrm{msnm}$. El periodo de estudio abarcó desde abril hasta agosto del 2002. Se seleccionaron 65 borregas Corriedale de 3 y 4 años de edad, de un total de 700 animales. Las ovejas tenían un peso promedio de 46.1 $\pm 4.5 \mathrm{~kg}$ al inicio del experimento y de $51.2 \pm$ $4.1 \mathrm{~kg}$ al servicio. Todos los animales fueron expuestos a las mismas condiciones de manejo, sanidad y alimentación.
El celo se sincronizó con esponjas vaginales impregnadas con $30 \mathrm{mg}$ de acetato de fluorogestona (FGA) y $0.175 \mathrm{mg}$ de Tiaprost, un análogo de prostaglandina $\mathrm{F}_{2 \propto}$, para inducir la regresión del cuerpo lúteo. Además se utilizaron carneros vasectomizados (retajos) para la detección del celo en las borregas.

El diseño experimental se describe en la Fig. 1. Se formaron tres grupos experimentales: GP10, GP4 y GC con 22, 20 y 23 animales, respectivamente. La sincronización del ciclo se inició en el día 0,6 y 12 días del inicio del experimento en el grupo GP10, GP4 y $\mathrm{GC}$, respectivamente. Cada hembra recibió intravaginalmente una esponja impregnada de $30 \mathrm{mg}$ de FGA por un periodo de 12 días. El día 0 del ciclo estrual fue el día en que las ovejas presentaron celo después del retiro de la esponja.

Las ovejas del GP4 y GP10 recibieron $0.75 \mathrm{mg}$ de una prostaglandina análoga (Tiaprost) en el día 4 y 10 del ciclo, respec-
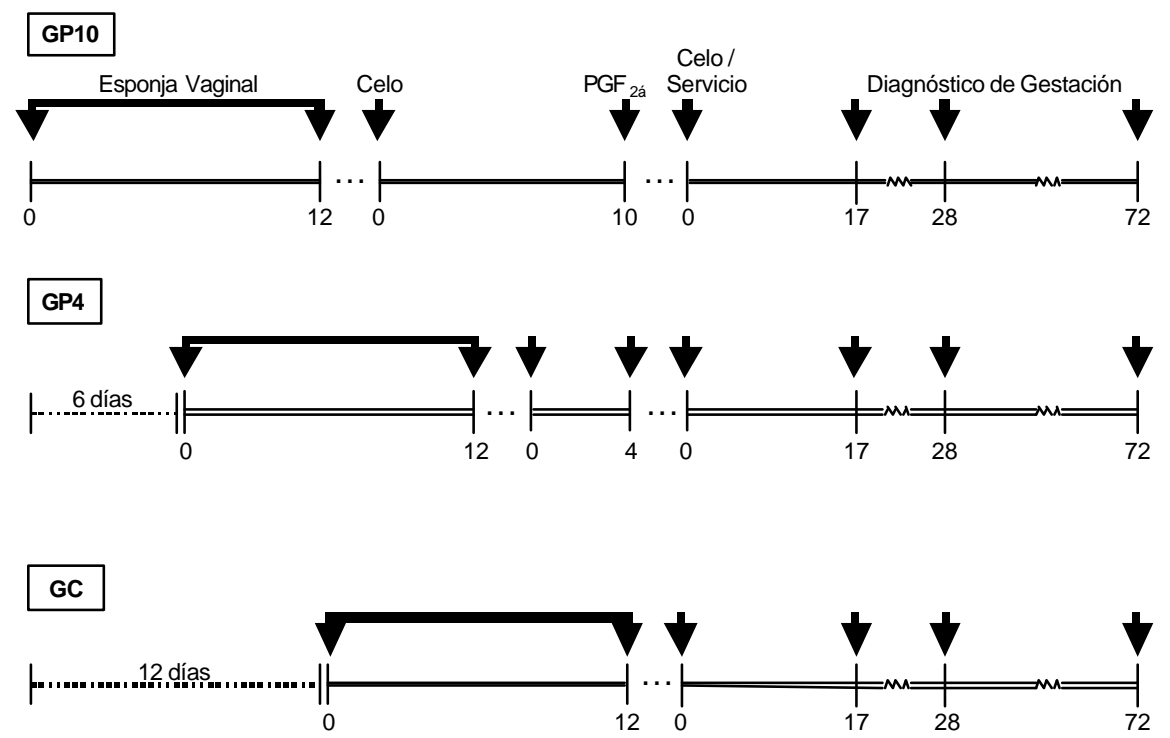

Figura 1. Secuencia experimental en el estudio de manipulación del folículo dominante con $\mathrm{PGF}_{2 \propto}$ en dos momentos del ciclo estrual (4 y 10 días después del celo) en borregas Corriedale 
tivamente. Ninguna oveja del GC recibió este tratamiento.

La detección de celo postsincronización y posttratamiento con prostaglandina se realizó en tres momentos del día (06:00, 12:00 y 17:00). Las ovejas en celo fueron separadas del grupo para facilitar la detección en el resto de las borregas.

Se registró la ocurrencia de celo, después de la sincronización con FGA, para determinar el día del ciclo estrual en que se administró la $\mathrm{PGF}_{2 \alpha}$. Asimismo, se registró la ocurrencia de celo después del tratamiento con PGF $_{2 \alpha}$ en GP4 y GP10, y se determinó el intervalo en que ocurrió después de la aplicación de $\mathrm{PGF}_{2 \alpha}$. Para fines comparativos, estos valores se compararon con el intervalo entre el retiro de la esponja vaginal y el celo en el GC.

El servicio en las ovejas en celo se realizó mediante monta directa, para lo cual se usaron seis carneros. El diagnóstico de preñez en las borregas se realizó a los 17 días después del servicio con el uso de retajos y a los 28 y 72 días mediante ultrasonografía transrectal, considerándose preñada con la observación del embrión.

Los análisis estadísticos empleados fueron aquellos del tipo "tiempo a un evento". El tiempo de la ocurrencia del celo después de la remoción de la esponja vaginal y la aplicación de la $\mathrm{PGF}_{2 \alpha}$ fueron analizados con la curva de supervivencia de Kaplan Meier. Las diferencias estadísticas entre las variables analizadas fueron determinadas mediante la Regresión de Cox. Los análisis de razón de riesgo (incidencia de celo y tasa de concepción) se efectuaron empleando regresión logística con tratamientos y carneros como variables predictoras.

\section{Resultados}

\section{Incidencia de celo post FGA}

El total de borregas de los tres grupos experimentales exhibió celo después del retiro de las esponjas intravaginales.

\section{Incidencia de celo postratamiento con PGF $_{2 \propto}$ e intervalo entre tratamiento $y$ presentación de celo}

La incidencia de celo en los días 4 (GP4) y 10 (GP10) del ciclo estrual fue del 100\%; sin embargo, hubo diferencia significativa en el intervalo entre administración de $\mathrm{PGF}_{2 \propto} \mathrm{y}$ la presentación de celo $(\mathrm{p}<0.01)$, siendo más temprano en GP4 respecto a GP10 y GC ${ }^{1}$ (Cuadro 1).

\section{Tasa de preñez}

La tasa de preñez a los 17 días $^{2}$ postservicio fue mayor en el GC que en los demás grupos experimentales; no obstante, estas diferencias no fueron significativas ( $p>0.05$, Cuadro 2). La tasa de preñez disminuyó en todos los grupos a los 28 días postservicio y se mantuvo hasta los 72 días con excepción de GC (Cuadro 2, p>0.05).

\section{Discusión}

Usualmente, en ovejas con cierta estacionalidad, y al inicio de la estación sexual después del anestro, ocurre el proceso de ovulación sin la presencia de celo en un gran porcentaje de los animales (Walton et al., 1977; Oldham y Martin, 1978). Sin embargo, en el presente estudio, no obstante que la fase experimental se inició en la última etapa del

${ }^{1}$ Intervalo entre el retiro de la esponja impregnada con FGA y la presentación de celo

${ }^{2}$ Diagnóstico de no retorno al celo mediante retajos (probable gestación) 
Cuadro 1. Incidencia de celo post tratamiento con $\mathrm{PGF}_{2^{\propto}}$ (día 10: GP10 y 4: GP4) y sin PG $\mathrm{F}_{2^{\infty}}(\mathrm{GC}$ : control) en ovinos Corriedale e intervalo entre el tratamiento y la presentación de celo

\begin{tabular}{|c|c|c|c|}
\hline Grupos & $\begin{array}{l}\text { Ovejas } \\
\text { (n) }\end{array}$ & $\begin{array}{l}\text { Incidencia de celo } \\
(\%)\end{array}$ & $\begin{array}{l}\text { Intervalo } \mathrm{PGF}_{2^{\propto}} \mathrm{y} \text { celo } \\
\quad\left(\text { promedio }^{2} \pm \mathrm{de}\right)\end{array}$ \\
\hline GP10 & 22 & 100 & $47: 45 \pm 7: 06^{\mathrm{a}}$ \\
\hline GP4 & 20 & 100 & $34: 28 \pm 4: 26^{\mathrm{b}}$ \\
\hline $\mathrm{GC}^{1}$ & 23 & 100 & $40: 16 \pm 6: 39 \mathrm{~b}$ \\
\hline Total & 65 & 100 & $41: 01 \pm 8: 10$ \\
\hline
\end{tabular}

Cuadro 2. Incidencia de celo post sincronización con PGF $_{2^{\propto}}$ (día 10: GP10 y 4: GP4) y sin $\mathrm{PGF}_{2 \alpha}$ (GC: control) en ovinos Corriedale y tasa de preñez a los 17, 28 y 72 días del servicio

\begin{tabular}{lcccccc}
\hline & \multicolumn{2}{c}{ GP10 } & \multicolumn{2}{c}{ GP4 } & \multicolumn{2}{c}{ GC } \\
\cline { 2 - 7 } & $\mathrm{n}$ & $\%$ & $\mathrm{n}$ & $\%$ & $\mathrm{n}$ & $\%$ \\
\hline Incidencia de celo $^{1}$ & 22 & 100.0 & 20 & 100.0 & 23 & 100.0 \\
\% preñez a 17 días & 17 & 77.3 & 16 & 80.0 & 21 & 91.3 \\
\% preñez a 28 días & 14 & 63.6 & 13 & 65.0 & 20 & 87.0 \\
\% preñez a 72 días & 14 & 63.6 & 13 & 65.0 & 18 & 78.3 \\
\hline
\end{tabular}

${ }^{1}$ El grupo GC fue con respecto a la esponja impregnada con FGA

${ }^{2}$ Diagnóstico de no retorno al celo mediante retajos (probable gestación)

anestro (abril) y el inicio de la estación sexual (mayo), el 100\% de ovejas tratadas con esponjas intravaginales mostró celo manifiesto. Este comportamiento sexual es el resultado del efecto sensibilizante del progestágeno (FGA) impregnado en la esponja vaginal sobre el hipotálamo para responder al efecto del estradiol $\left(\mathrm{E}_{2}\right)$ del folículo preovulatorio (Legan y Karsch, 1979; Legan et al., 1985). Similares resultados con el uso de otro progestágeno (MAP) son reportados por Cárdenas (1997) y Oyediji et al. (1990), en contraste con otros trabajos que reportan un menor efecto con el uso de MAP en ovejas Corriedale (Iglesias et al., 1997) y con FGA en ovejas Dorset (Rajamahendran et al., 1993).

Estas diferencias podrían ser explicadas por el periodo de anestro en que se encontraban las ovejas al momento del tratamiento, siendo la respuesta menos marcada cuando el animal se encuentra en periodo de anestro profundo (Thomas et al., 1988; Smith et al., 1988) y por fallas en la detección de celos de corta duración por menor frecuen- 
cia de chequeo (Smith et al., 1987, 1988; Robinson, 1971, 1980; Ginther et al., 1995). En el presente estudio, la eficiencia en la detección del celo estuvo asociada a la frecuencia de chequeo del celo por día $(6,12$ y 17 h) $\mathrm{y}$ al uso de machos retajos con marcadores.

$\mathrm{La} \mathrm{PGF}_{2 \alpha}$ originó la regresión del cuerpo lúteo en todas las borregas (McCracken et al., 1972; Boland et al., 1978; Knickerbocker et al., 1988; Niswender y Nett, 1988). Se esperaba encontrar que las ovejas tratadas con $\mathrm{PGF}_{2 \alpha}$ en el día 4 (GP4) postcelo no presentasen signos de celo, de acuerdo a los hallazgos de Acritopoulou y Haresing (1980), Wiltbank y Niswender (1992), Hansel y Convey (1983), Deaver et al. (1986) y Hafez (1989). Estos autores reportan que la $\mathrm{PGF}_{2 \alpha}$ no afecta el desarrollo y vida del cuerpo lúteo cuando es administrada antes del día 5 post-celo, asumiendo este hecho a la ausencia o escasa presencia de receptores de $\mathrm{PGF}_{2 \alpha}$ en las células luteales (Wiltbank et al., 1995). Sin embargo, es más probable que este hecho ocurra entre los días 0 y 3 postcelo ( 1 ó 2 postovulación), considerando el hallazgo reportado por Leyva (1996) y Leyva et al. (1998b) al monitorear los cambios de los niveles de progesterona $\left(\mathrm{P}_{4}\right)$, y observar la regresión prematura del cuerpo lúteo entre los días 4 y 5 postcelo en ovejas en fase de anestro final que fueron inducidas a ovular conPMSG.

Se sugiere como responsable de la regresión del cuerpo lúteo al $\mathrm{E}_{2}$ de folículos mayores de $7 \mathrm{~mm}$ de diámetro desarrollados antes y después de la ovulación (Leyva et $a l ., 1998 \mathrm{a}, \mathrm{b})$ al estimular la síntesis de receptores de oxitocina (Roberts et al., 1975, 1976; McCracken et al., 1984) y secreción de prostaglandina (Vallet et al., 1990), hormonas que intervienen en el proceso luteolítico (Schams y Kartg, 1982; Flint y Sheldrick, 1983; Fitz et al., 1982; Knickerbocker et al., 1988; Niswender y Nett, 1988; Tsai y Wiltbank, 1988; Wiltbank y Niswender, 1992). Hay estudios que reportan suficiente presencia de receptores de oxitocina y prostaglandina en el CL para res- ponder al efecto de la $\mathrm{PGF}_{2 \alpha}$ exógena entre los días 3 y 5 del ciclo estrual (Wiltbank et al., 1995).

La ocurrencia de celo más temprana en borregas tratadas con $\mathrm{PGF}_{2 \alpha}$ en el día 4 (34:28 $\pm 4: 26 \mathrm{hs}$ ) que en aquellas tratadas en el día $10(47: 45 \pm 7: 06 \mathrm{~h})$, es comparable a los reportes de Deaver et al. (1986) en los días 5 $(39 \pm 1 \mathrm{~h}), 8(49 \pm 6 \mathrm{~h})$ y $11(58 \pm 7 \mathrm{~h}) \mathrm{del}$ celo, y de Acritopoulou y Haresing (1980) en los días $8(39.8 \pm 2 \mathrm{~h})$ y $11(45.7 \pm 1.5 \mathrm{~h})$ del ciclo.

Los resultados del presente estudio, así como los reportados por otros autores en bovinos, sugieren y corroboran que la ocurrencia de celo es más temprana cuando la $\mathrm{PGF}_{2 \alpha}$ induce la regresión del CL en estadios más tempranos de su desarrollo. En este estudio no hubo seguimiento ecográfico del desarrollo de la actividad folicular y, por lo tanto, no se pudo determinar a qué onda pertenecieron los folículos ovulatorios; sin embargo, se podría inferir en base a los estudios ecográficos de la dinámica folicular reportados por Leyva (1996) y Leyva et al. (1998 a,b) que para GP4 fue la primera onda folicular y para GP10 la segunda o tercera onda folicular.

En el día 4 del ciclo, el folículo dominante de la primera onda folicular y el CL se encuentran en fase de crecimiento (Leyva $e t$ al., 1998b); próximos a alcanzar su máximo desarrollo, lo que se refleja en la secreción de $\mathrm{P}_{4}$; mientras que en el día 10 del ciclo, el CL está en su máximo desarrollo con su máxima producción de $\mathrm{P}_{4}$ y ejerciendo su máximo efecto inhibitorio (Leyva, 1996; Leyva et al., 1998b). Estas diferencias entre el menor efecto inhibitorio de $\mathrm{P}_{4}$ en el día 4 y el máximo efecto en el día 10 del crecimiento folicular, guarda relación con la inhibición de la secreción pulsátil de LH, siendo menor en el día 4 y mayor en el día 10, lo cual concuerda, además con el mayor tamaño del folículo dominante de la primera onda folicular que el de la segunda y tercera onda folicular. Este análisis permite inferir una pérdida más rápida 
del efecto inhibitorio de los niveles crecientes de $\mathrm{P}_{4}$ por efecto de la $\mathrm{PGF}_{2 \alpha}$ aplicada en el día 4 del ciclo, con el resultado de un crecimiento rápido del folículo ovulatorio; lo cual explicaría en parte el mayor intervalo entre el momento de la administración de $\mathrm{PGF}_{2 \alpha} \mathrm{y}$ la ocurrencia del celo en GP10 respecto a GP4; así como también la menor variabilidad entre borregas en la presentación del celo de GP4 respecto a GP10.

En el grupo control, al ser un grupo de borregas escogidas al azar y sin conocimiento del momento del ciclo estrual en el que se les insertó la esponja, se esperarían dos estadios sobre la dinámica folicular (Leyva, 1996); uno supresor (coincidiendo con la fase luteal con niveles de $\mathrm{P}_{4}>1 \mathrm{ng} / \mathrm{ml}$ ) y otro estimu-latorio (con niveles de $\mathrm{P}_{4}$ bajos o basales: $<1 \mathrm{ng} / \mathrm{ml}$ ). Estos efectos ocasionan una decreciente y creciente secreción pulsátil de LH, respectivamente (Savio et al., 1993); consecuentemente afectando el desarrollo folicular y la respuesta del eje hipotálamo-hipófisis, determinando que un grupo de borregas entraran en celo tardíamente y otras más temprano.

No se pudo determinar si todas las borregas que presentaron celo luego de la aplicación de la $\mathrm{PGF}_{2 \alpha}(\mathrm{GP} 10$ y GP4) y retiro de esponja (GC) ovularon y lograron ser fertilizadas luego de la monta. Sin embargo, la tasa de no retorno al celo o probable preñez a los 17 días del servicio estuvo en el rango de 77 a $91 \%$. La ausencia de preñez en el resto de animales fue probablemente debido a fallas en la ovulación, fallas en la fecundación o una temprana mortalidad embrionaria ocurrida antes de la regresión del cuerpo lúteo. Estos resultados son mas eficientes a los reportados por Hanly (1961), Perry y Rowlands (1962) y Sreenan y Diskin (1986), quienes manifiestan una mortalidad embrionaria temprana normal entre 20 a $40 \%$.

Si bien es cierto que no existieron diferencias estadísticas en tasa de preñez entre los grupos tratados con prostaglandinas (GP4 y GP10) y el control (GC), se observó un mayor porcentaje de retorno al celo en bo- rregas del GP10 y GP4 con respecto a GC. Woody et al. (1967), Ottobre et al. (1980) y Pope et al. (1995) mencionan que para la secreción de $\mathrm{PGF}_{2 \alpha}$ se requiere de un tiempo mínimo de exposición del endometrio a concentraciones relativamente altas de progesterona. En el presente trabajo, el endometrio de los animales de GP4 y GP10 tuvo menor tiempo de exposición a $\mathrm{P}_{4}$, lo que sería una probable desventaja para estos grupos, resultando en una relativa menor fertilidad. Por otro lado, Razdan et al. (2001) reportan que la $\mathrm{PGF}_{2 \alpha}$ afecta la motilidad del oviducto, y por consiguiente el transporte del ovocito o embrión, el número de espermatozoides que acceden a la zona pelúcida, y el proceso de división embrionaria.

Durante el ciclo estrual, la fase luteal ocupa el mayor tiempo (ovulación a luteólisis) y la fase folicular un menor tiempo (luteólisis a ovulación). De acuerdo a esto, se podría asumir que cuando se insertaron las esponjas vaginales al grupo control (GC), un pequeño número de estas ovejas estarían en fase folicular, donde los niveles de $\mathrm{P}_{4}$ endógeno son bajos. Según Leyva (1996), estos niveles parecen ser la causa del persistente incremento (por el efecto subluteal) en el crecimiento del folículo mayor en los tratamientos largos de progestágeno (12 días), que al retirarse favorecería la ovulación de folículos con vida media prolongada (Viñoles et al., 2001). Esto sustenta la hipótesis que estos tratamientos promueven la ovulación de ovocitos viejos que al ser fertilizados, el embrión resultante tiene poca probabilidad de sobrevivir debido a un desarrollo anormal. Esto explicaría el incremento de mortalidad embrionaria a los 72 días postservicio.

Miller y Moore (1976) indican que en estadios previos del blastocisto, se desarrollan factores tales como la calidad de la ovulación folicular y la magnitud de luteinización, determinando el rango de la secreción luteal de $\mathrm{P}_{4}$. Además, en rumiantes, la pérdida embrionaria ocurre cuando la $\mathrm{P}_{4}$ que corresponde a un ciclo anterior o el estrógeno que corresponde al estro se omite, afectando el 
tiempo de inicio del mecanismo luteolítico. Esto probablemente permite explicar que algunos de los embriones de GP4 y GP10, a los que antecedieron ciclos cortos (4 y 10 días) serían afectados en su desarrollo, no permitiendo una buena coordinación madre-embrión (señales inadecuadas de embriones débiles para indicar su presencia en el momento apropiado) (Demmers et al., 2001), pero que permitiría un tiempo de vida ligeramente mayor del CL, dando lugar probablemente a la muerte embrionaria alrededor y posterior del reconocimiento maternal de la preñez. Esto se manifestaría en un mayor número de animales no gestantes a los 28 días, verificados mediante la presencia del embrión en estos grupos, respecto a GC.

\section{Conclusiones}

- Todas las borregas presentaron celo a la sincronización con esponja intravaginal (FGA).

- Todas las borregas presentaron celo al tratamiento con prostaglandina $\mathrm{F}_{2 \alpha}$

- El celo en borregas después de la aplicación de prostaglandina $\mathrm{F}_{2 \alpha}$ en el día 4 del ciclo estrual ocurrió más temprano (34:28 h), que en aquellas tratadas en el día $10(47: 45 \mathrm{~h})(\mathrm{p}<0.01)$.

- Las borregas sincronizadas con prostaglandina $\mathrm{F}_{2 \alpha}$ en el día 4 del ciclo presentaron menor variabilidad ( $\mathrm{de}=4: 26 \mathrm{~h}$ ) a la presentación del celo que las sincronizadas el día 10 (de = 7:06 h).

- La tasa de preñez (no retorno al celo) a los 17 días postservicio fue similar en ambos grupos tratados con prostaglandina $\mathrm{F}_{2 \alpha}$; sin embargo, porcentualmente fueron menores que el control $(77.3,80.0$ y $91.3 \%$ para GP10, GP4 y GC, respectivamente); reduciéndose la tasa de gestación a los 28 días en todos los grupos (63.6, 65.0 y 87.0, respectivamente), y manteniéndose hasta los 72 días con excepción de GC (78.3\%).

\section{Literatura Citada}

1. Acritopoulou, S.; W. Haresing. 1980. Reponse of ewes to a single injection of an analogue of $\mathrm{PGF}_{2 \alpha}$ given at different stages of the oestrus cycle. J. Reprod. Fert. 58: 219-223.

2. Boland, M.P.; I. Gordon; D.C. Keller. 1978. The effect of treatment by prostaglandin analogue (I.C.I. 80996) or progestagen (SG-9880) on ovulation and fertilization in cyclic ewes. J. Agric. Sci., Camb. 91: 727-730.

3. Cárdenas, H.; K.E. McClure; W.F. Pope. 1993. Luteal function and blastocyst development in ewe following treatment with $\mathrm{PGF}_{2 \alpha}$ and GnRH. Theriogenology 40: 865 .

4. Cárdenas, H. 1997. Control artificial del ciclo estral en ovinos. Memoria I Symposium Internacional: Avances en Reproducción de Rumiantes. p 99-105. APPA.

5. Champa, L. 2000. Efecto de la ovulación del folículo dominante del día 7 y 13 del ciclo estral con $\mathrm{PGF}_{2 \alpha}$ sobre las tasas reproductivas en vacas. Tesis de Médico Veterinario. Facultad de Medicina Veterinaria, Univ. Nacional Mayor de San Marcos. Lima. 56 p.

6. Deaver D.R.; N.J. Stilley; R.A. Dailley; E.K. Inskeep; P.E. Lewis. 1986. Concentration of ovarian and pituitary hormones following prostaglandin $\mathrm{F}_{2 \alpha}$-induced luteal regression in ewes varies with day of estrous cycle at treatment. J. Anim. Sci. 62: 422-427.

7. Demmers, K.J.; K. Derecka; A. Flint. 2001. Trophoblast interferon and pregnancy. Reproduction 121: 41-49.

8. Fitz, T.A.; M.H. Mayan; H.R. Sawyer; G.D. Niswender. 1982. Characterization of two steroidogenic cell types in the ovine corpus luteum. Biol. Reprod. 27: 703-711.

9. Flint, A.P.F.; E.L. Sheldrick. 1983. Evidence for a systemic role for ovarian oxytocin in luteal regression in sheep. J. Reprod. Fert. 67: 215-225. 
10. Ginther, O.J.; K. Kot; M.C. Wiltbank. 1995. Associations between emergence of follicular waves and fluctutations in FSH concentrations during the oestrus cycle in ewes. Theriogenology 43: 639703.

11. Ginther, O.J.; J.P. Kastelic; L. Knopf. 1989. Composition and characteristics of follicular waves during the bovine estrous cycle. Anim Reprod. Sci. 20:187-200.

12. Hafez, E.S.E. 1989. Reproducción e inseminación artificial en animales domésticos. $5^{\mathrm{a}}$ ed. Nueva Editorial Interamericana. México. $694 \mathrm{p}$.

13. Hafez, E.S.E. 1993. Reproducción e inseminación artificial en animales. $6^{\mathrm{a}}$ ed. Interamericana McGraw Hill. México $543 \mathrm{p}$.

14. Hanly, S. 1961. Prenatal mortality in farm animals. J. Reprod. Fertil. 2: 182194.

15. Hansel, W.; E. Convey. 1983. Phisiology of the estrous cycle. J. Anim. Sci. 37: 404-424.

16. Iglesias, R.M.R.; N.H. Ciccioli; H. Irazoqui. 1997. Ram-induced reproduction in seasonally anovular Corriedale ewes: MAP doses for oestrous induction, ram percentages and post-mating progestagen supplementation. Anim. Sci. (Pencaitland) 64: 119-125.

17. Illera, M. 1994. Reproducción de los animales domésticos. Ed. Aedos. Barcelona, España. 390 p.

18. Knickerbocker, J.J.; M.C. Wiltbank; G.D. Niswender. 1988. Mechanisms of luteolysis in domestic livestock. Dom. Anim. Endocrinol. 5: 91-107

19. Legan, S.J.; F.J. Karsch. 1979. Neuroendocrine regulation of the oestrus cycle and seasonal breeding in the ewe. Biol. Reprod. 20: 74-85.

20. Legan, S.J.; H. I'Anson; B.P. Fitzgerald; M.S. Akaydin. 1985. Importance of short luteal phases in the endocrine mechanism controlling initiation of estrous cycles in anestrous ewes. Endocrinology 117: 1530-1536.

21. Leyva, V. 1996. Follicular activity and ovulation of ewes during the breeding season and anestrus. Ph.D. Thesis. Guelph University. Canada. 278 p.

22. Leyva, V.; B.C. Buckrell; J.S. Walton. 1998a. Follicular activity and ovulation regulated by exogenous progestagen and PMSG in anestrous ewes. Theriogenology 50: 377-393.

23. Leyva, V.; B.C. Buckrell; J.S. Walton. 1998b. Regulation of follicular activity and ovulation in ewes by exogenous progestagen. Theriogenology 50: 395-416.

24. McCracken, J.A.; J.C. Carlsson; M.E. Glew; J.R. Goding; D.T. Baird; K. Green; B. Samuelsson. 1972. Prostaglandin $\mathrm{F}_{2 \propto}$ identified as a luteolytic hormone in sheep. Nature, New Biol. 238:129-134.

25. McCracken, J.A.; W. Schramm; W.G. Okulicz. 1984. Hormone receptor control of pulsatile secretion de $\mathrm{PGF}_{2 \propto}$ from the ovine uterus during luteolysis and its abrogation during early pregnancy. Anim. Reprod. Sci. 7: 31-35.

26. Miller, B.G.; N.W. Moore. 1976. Effects of progesterone and oestradiol on endometrial metabolism and embryo survival in the ovariectomized ewe. Theriogenology 6: 636.

27. Niswender, G.D.; T.M. Nett. 1988. The corpus luteum and its control. En: E. Knobil et al. (eds). The Physiology of Reproduction. p 489-525. Raven Press. New York.

28. Oldham, C.M.; G.B. Martin. 1978. Stimulation of seasonally anovular Merino ewes by rams. II. Premature regression of ram induced corpora lutea. Anim. Reprod. Sci. 1: 291-295.

29. Ottobre, J.S.; G.S. Lewis; W.V. Thayne; E.K. Inskeep. 1980. Mechanism by which progesterone shorten the duration of the estrous cycle of the ewe. Biol. Reprod. 23: 1046-1053.

30. Oyediji, G.O.; M.O. Akusu; G.N. Egbunike. 1990. Comparative studies on the effectiveness of sil-estrus implants, Veramix sheep sponges and prostaglandin F-2-alpha in synchronizing estrus in West African Dwarf sheep. Theriogenology 34: 613-618. 
31. Perry, J.S.; I.W. Rowlands. 1962. Early pregnancy in the pig. J. Reprod. Fertil. 4:175-188.

32. Pope, W.F.; H. Cardenas; T.M. Wiley; K.E. McClure. 1995. Dose-response relationship of exogenus progesterone shortly after ovulation on estrous cycle length, blastocyst development and fertility in sheep. Anim. Reprod. Sci. 38: 109-117.

33. Rajamahendran, R.; J. Raniowski; V. Ravindran. 1993. Effect of PMSG and ram contact on the reproductive performance of progestagen-treated ewes during breeding and anestrous seasons. Small Rumin. Res. 10: 341-347.

34. Razdan, P.; A.M. Mwanza; H. Kindahl; F. Hulten; S. Einarsson. 2001. Impact of postovulatory food deprivation on the ova transport, hormonal profiles and metabolic changes in sows. Acta Vet. Scand. 42: 15-25.

35. Roberts, J.S.; B. Barcikowski; L. Wilson; R.C. Skarnes; J.A. McCraken. 1975. Hormonal and related factors afecting the release of prostaglandin $\mathrm{F}_{2 \alpha}$ from the uterus. J. Steroid. Biochem. 6: 1091.

36. Roberts, J.S; J.A. McCracken; J.E. Gavagan; M.S. Soloff. 1976. Oxytocinstimulated release of prostaglandin $\mathrm{F}_{2 \alpha}$ from bovine endometrium in vitro: correlation with estrous cycle and oxytocin-receptor binding. Endocrinology 99: 1107-1114.

37. Robinson, T.J. 1971. The seasonal nature of reproductive phenoma in sheep. II. Variation in fertility following fertilization of oestrus. J. Reprod. Fert. 24: 19-27.

38. Robinson, T.J. 1980. Programmed year-round sheep breeding. Aust. J. Exp. Agric. Anim. Husb. 20: 667-673.

39. Rubianes, E.; R. Ungerfeld; C. Viñoles; A. Rivero; G.P. Adams. 1997a. Ovarian response to gonadotropin treatment initiated relative to wave emergence in ultrasonographically monitored ewes. Theriogenology 47: 1479-1488.

40. Rubianes, E.; A. Beard; D.J. Dierschke; P. Bartlewski; G.P. Adams; N.C. Rawlings. 1997b. Endocrine and ultrasound evaluation of the response to induced ovulation at different stages of the luteal phase in cycling ewes. Theriogenology 48: 1093-1104.

41. Savio J.D.; L. Keenan; M.P. Boland; J.F. Roche. 1988. Pattern of growth of dominant follicles during the oestrus cycle in heifers. J. Reprod. Fert. 88: 663-671.

42. Savio, J.D.; W.W. Thatcher; G.R. Morris; K. Entwistle; M. Drost; K. Mattiacci. 1993. Effects of induction of low plasma progesterone concentration with a progesterone releasing intravaginal device on follicular turnover and fertility in cattle. J. Reprod. Fert. 98: 77-84.

43. Schams, D.; H. Kartg. 1982. Hormonal responses following treatment with different prostaglandin analogues for estrous cycle regulation in cattle. Prostaglandins 17: 499-513.

44. Smith, J.F.; L.T. McGowan; K.S. Maclean; P.A. Farquhar. 1987. The effect of steroid immunization on the seaonal pattern of oestrus, ovulation and ovulation rate in Coopworth ewes. Proc. N. Z. Soc. Anim. Prod. 47: 143-146.

45. Smith, J.F.; G.J. Cruickshank; L.T. McGowan; J. Parr; B.J. Mortimer. 1988. Seasonal changes in oestrus, ovulation and conception of Coopworth ewes treated with CIDRs and PMSG. Proc. N. Z. Soc. Anim. Prod. 48: 99102.

46. Sreenan, J.M.; M.G. Diskin. 1986. The extent and timming of embryonic mortality in the cow. En: Embryonic mortality in farm animals. J.M.Sreenan; M.G.Diskin (eds). Boston Martinus Nijhoff.

47. Thomas, G.B.; D.T. Pearce; C.M. Oldam; G.B. Martin; D.R. Lindsay. 1988. Effects of breeds, ovarian steroids and season on the pulsatile secretion of 
LH in ovariectomized ewes. J. Reprod. Fert. 84: 313-324.

48. Tsai, S.; C. Wiltbank. 1988. Prostaglandin $\mathrm{F}_{2 \alpha}$ regulates distinct physiological changes in early and midcycle bovine copora lutea. Biol. Reprod. 58: 346-352.

49. Vallet, J.L.; G.E. Lamming; M. Batten. 1990. Control of endometrial oxytocin receptor and uterine response to oxytocin by progesterone and estradiol in the ewe. J. Reprod. Fert. 90: 625-634.

50. Viñoles, C.; M. Fosberg; G. Banchero; E. Rubianes. 2001. Effect of long-term and short-term progestagen treatment on follicular development and pregnancy rate in cyclic ewes. Theriogenology 55: 993-1004.

51. Walton, J.S.; J.R. McNeilly; N.S. McNeilly; F.J. Cunningham. 1977. Changes in concentration of follicle esti- mulating hormone, luteinizing hormone, prolactin and progesterone in the plasma of ewes during the transition from anoestrus to breeding activity. J. Endocrinol. 75: 127-136.

52. Wiltbank, M.; J. Niswender. 1992. Functional aspects of differentiation and degeneration of steroidogenic cells of corpus luteum in domestic ruminants. Anim. Reprod. Sci. 28: 103-110.

53. Wiltbank, M.C; T.F. Shiao; D.R. Bergfelt; O.J. Ginther. 1995. Prostaglandin F2 alpha receptors in the early bovine corpus luteum. Biol. Reprod. 52: 74-78.

54. Woody, C.O.; N.L. First; A.L. Pope. 1967. Effects of exogenous progesterone on estrous cycle length. J. Anim. Sci. 26: 139-141. 